This is an electronic reprint of the original article. This reprint may differ from the original in pagination and typographic detail.

Author(s): Jiang, Fan; Käkölä, Timo

Title: Wireless ATM : handover issues

Year: $\quad 1998$

Version:

Please cite the original version:

Fan Jiang; Käkölä, T., "Wireless ATM: handover issues". In: Personal, Indoor and Mobile Radio Communications, 1998. The Ninth IEEE International Symposium on , vol.1, no., pp.272-277 vol.1, 8-11 Sep 1998. doi: 10.1109/PIMRC.1998.733558

All material supplied via JYX is protected by copyright and other intellectual property rights, and duplication or sale of all or part of any of the repository collections is not permitted, except that material may be duplicated by you for your research use or educational purposes in electronic or print form. You must obtain permission for any other use. Electronic or print copies may not be offered, whether for sale or otherwise to anyone who is not an authorised user. 


\section{Wireless ATM: Handover Issues}

Fan Jiang

\author{
Nokia Telecommunications Oy, \\ Torikatu 6, FWN-44100, Äänekoski, Finland \\ Fan.Jiang@ntc.nokia.com
}

\begin{abstract}
Basic aspects of cellular systems and the ATM transmission technology are introduced. Wireless ATM is presented as a combination of Radio ATM and Mobile ATM. Radio ATM is a wireless extension of an ATM connection while Mobile ATM contains the necessary extensions to ATM to support mobility.

Because the current ATM technology does not support mobility, handover becomes one of the most important research issues for wireless ATM. Wireless ATM handover requirements are thus analysed. A handover solution relying on two inter-related handover protocols is presented and evaluated. Future research is needed to compare this solution with options presented by earlier research and possibly combine their best features to yield even better solutions.
\end{abstract}

\section{INTRODUCTION}

The introduction of second generation digital cellular systems has brought people to a new era. Communication has become easier and more mobile. But digital cellular systems are limited in their capacity to provide multimedia services. Consequently, there is a huge global market demand for a third generation mobile network that can provide multimedia services effectively. To meet this demand, the third generation mobile network must realise wireless ATM (WATM), that is, combine ATM and mobile technologies.

ATM (Asynchronous Transfer Mode) is another recent "hot" technology. Characterised by digitised 53 octets cell transmission, ATM takes streams of digital information (video, audio, and data) and transports them across the network simultaneously. Due to its high speed (10 Gbit/s and higher) and cell structure, ATM provides an efficient way of carrying multimedia applications with low delay and guaranteed quality of service (QoS).

The third generation mobile network will be a huge breakthrough. Adding mobility to the current ATM network is a complex undertaking and provides many areas for further research. The WATM handover concept (hereafter "Handover") is one of the most important areas to be explored.

This paper briefs some concepts about ATM and wireless networks to pave the way for further development of Handover. The paper contributes to current research in WATM principles
Timo Käkölä

\author{
University of Jyväskylä \& Turku Centre for Computer Science \\ FIN-40351 Jyväskylä, Finland \\ Timo.Kakola@cc.jyu.fi
}

by analysing requirements for Handover design and by proposing and explaining a Handover solution that combinestwo WATM handover protocols to meet the requirements.

In Chapter II, the architecture, connection, and QoS of ATM are explained. In Chapter III, wireless networks are discussed. The architecture and handover design of GSM (Global System for Mobile Communications), one of the most successful second generation digital cellular systems, is explained. Chapter IV first defines WATM and briefs its protocols. The handover requirements of WATM are then analysed. A hybrid of two WATM handover protocols is proposed and evaluated in Chapter V. In Chapter VI, conclusions are stated and issues for future research are proposed.

\section{U. ATM NETWORK}

The emergence of the Broadband Integrated Services Digital Network (B-ISDN) vagues the boundary between isochronous (audio and video) and asynchronous (data) services. ATM has been recommended by the international standardisation organisation ITU-T to be applied in B-ISDN. ATM is also accepted globally by industry. It is an innovation that could support voice, data and video services interchangeably. The conventional isochronous and asynchronous services can be requested by specifying the type of service from service categories including: (a) constant bit rate (CBR); (b) real-time variable bit rate (rt-VBR); (c) non-real-time variable bit rate (nIt-VBR); (d) unspecified bit rate (UBR); and (e) available bit rate $(A B R)$.

\section{A. Native-layered ATM Architecture}

Figure 1 shows the layered architecture of an ATM network as specified in the B-ISDN protocol reference model of ITU-T Recommendation I.321 [1, p. 2]. Each layer provides services to the next higher layer and receives services from the next lower layer in a way similar to that of the OSI (Open Systems Interconnection) model defined by ISO (International Standards Organisation). The physical layer defines the underlying mechanisms for delivering ATM cell streams through physical medium. The ATM layer performs cell switching and multiplexing functions. The ATM adaptation layer (AAL) adapts the services provided by the ATM layer to the individual needs of the higher layer applications, defines a set of service classes in order to accommodate different needs of users, and provides segmentation and re-assembly functions. 


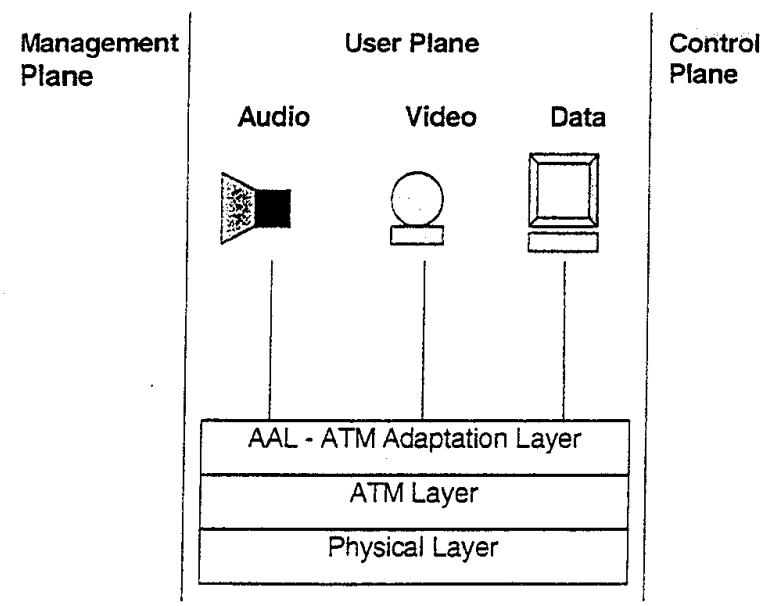

Figure 1. The Native-Layered ATM Architecture.

\section{B. ATM Connections}

ATM is a connection-oriented transmission technology. During the transmission, cells are transferred through the network via a fixed route. Unlike connectionless data networks, ATM preserves the sequence of the cells.

The building blocks of ATM networking are the transmission path, the Virtual Path (VP), and the Virtual Channel (VC). A transmission path contains one or more virtual channels (VC). Multiple virtual channels can be trunked to a single virtual path (VP) [2, p. 287].

\section{ATM supports these connections:}

(1) Switched Virtual Connection;

(2) Semi-Permanent Virtual Connection; and

(3) Permanent Virtual Connection (PVC).

There are two ATM connection types: point-to-point (unicast) and point-to-multipoint (multicast). Unicast can be set as unidirectional or bidirectional. Multicast connections transmit data only unidirectionally from the root to all leaves concerned. In this case, ATM switches simply replicate cells as necessary and distribute them to all the required destinations.

\section{Quality of Service-Classes}

QoS-classes are defined by the ATM Forum to fulfil users' requirements on a per-ATM-connection basis. Multiple Virtual Channel links, each with a different QoS-class, can be transported in the same virtual path connection (VPC).

The ATM Forum has classified QoS classes available to the Virtual Path and Virtual Channel connection options as Specified QoS (most stringent \& most versatile) and Unspecified QoS (less stringent \& expensive).

For Specified QoS-class, service providers must meet or surpass agreed
Maximum Cell Delay Time,

Maximum Cell Delay Variation,

Maximum Cell Loss Rate, and

Maximum Burst Cell Loss.

For Unspecified QoS-class, no network performance parameters are specified. However, the network provider may determine a set of internal objectives for the performance parameters [3, $p$. 7].

\section{WIRELESS NETWORKS}

The first generation analogue cellular mobile communication systems such as NMT (Nordic Mobile Telecommunications), AMPS (Advanced Mobile Phone System), and TACS (Total Access Cellular System) were introduced in early 1980s. In 1990s, the second generation digital cellular systems like GSM and DECT (Digital European Cordless Telephone) emerged. GSM has been very successful in recent years. In the following, we use GSM to illustrate basic concepts of wireless networks.

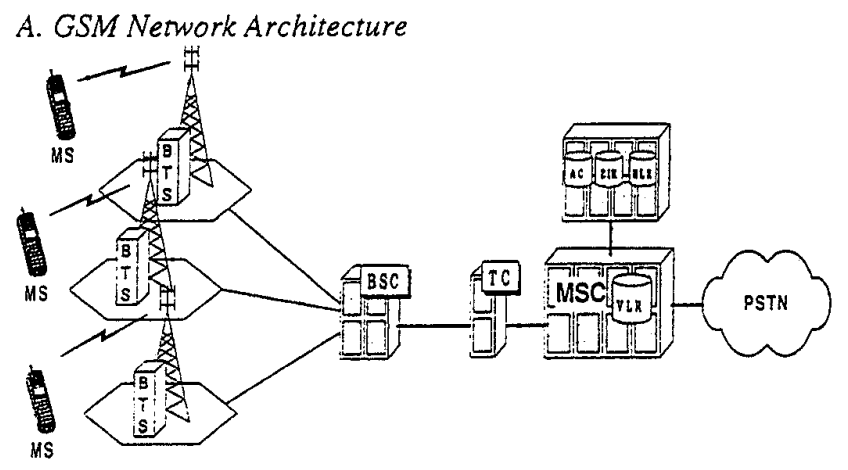

Figure 2. GSM network architecture $[4$, p. 701$]$.

Figure 2 shows the GSM network architecture. A cellular network elements include: (1) Base Transceiver Stations (BTS); (2) Base Station Controller (BSC); (3) TransCoder (TC); (4) Mobile Switching Center (MSC); (5) Home Location Register (HLR); (6) Visitor Location Register (VLR); (7) Authentication Center (AC); and (8) Equipment Identity Register (EIR). A set of BTSs is connected directly to BSC and through TC to MSC. Finally, MSC connects the lines to a public switched telephone network (PSTN).

Mobility Management is very important for GSM networks because it enables a Mobile Station (MS) to move freely in both the GSM Home Network and GSM Visited Network. Procedures included in Mobility Management are Location Registration, Location Update, Paging, and Handovers. In the following, we discuss GSM handover protocols.

\section{B. GSM Handover}

Handover occurs when a Mobile Station changes either signalling or a traffic channel, that is, from the view point of the 
network, the MS is handed over to the next BTS. There are four types of handovers in GSM:

(1) Intracell (Channel to Channel) Handover - MS changes a traffic channel inside the BTS (wireless cell);

(2) Intercell, IntraBSC, BTS to BTS Handover - MS transfers a call between wireless cells under the control of the same Base Station Controller (BSC);

(3) Intercell, InterBSC, BSC to BSC Handover - MS transfers a call between wireless cells under the control of different BSCs but belonging to the same Mobile Switching Centre (MSC);

(4) Intercell, InterMSC, MSC to MSC Handover - MS transfers a call between wireless cells under the control of different MSCs.

GSM handovers are performed by the network. It partitions the handover tasks and localises the handover traffic with respect to locality and the types of migration. GSM systems also support the transfer of handover control functions from one network entity to another. This transfer capability coupled with a personal identity concept have made international roaming possible, hence achieving both terminal and personal mobility.

\section{WIRELESS ATM (WATM)}

Second generation digital cellular systems are very successful. But global markets will soon arise for the third generation cellular systems because there is an ever increasing demand for high bit rate services enabling, for example, simultaneous video, audio, and data transmission. An integration of ATM transmission and wireless network technologies is crucial for meeting these needs effectively.

The WATM concept extends ATM into the wireless environment. Mobility and radio aspects are introduced into the ATM transmission. In general, WATM means the combination of Mobile ATM and Radio ATM (Figure 3). Mobile ATM is an extension of normal ATM that includes support for mobility (e.g., handovers). Radio ATM is a wireless extension of an ATM connection (Virtual Channels over radio). Wireless ATM is an end-to-end ATM connection including a wireless link.

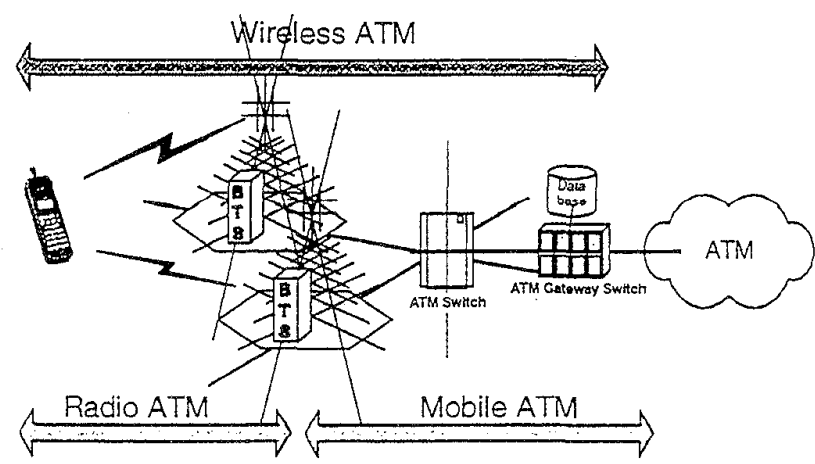

Figure 3. General Concept of WATM [5, p. 4].
In the following sections we first present WATM protocols anc then discuss the requirements for a seamless handover in a WATM environment. In the next chapter, we explain and evaluate a hybrid of two WATM handover protocols that meets these requirements.

\section{A. WATM Protocols}

WATM needs to support: (a) fast handover; (b) high bandwidth; and (c) mobile quality of service. Since a Wireless ATM network is an extension of the wired ATM network using a "wireless-last-hop" from BTS to MS, the control and management functions of wired ATM will still be present. In addition to these functions, WATM protocols include:

(1) Mobile Connection Management Protocol (MCMP)

MCMP describes how a user can initiate a mobile call and specify the desired QOS and how the network can support the QOS and reserve resources throughout the duration of the call. It also governs the allocation and de-allocation of VCs over both wired and wireless links. MCMP must also allow the establishment of partial or new paths during handovers.

(2) Mobile Handover Management Protocol (MHMP) MHMP describes how on-going calls can be supported when mobile users are roaming. It has the following steps: (a)? determining the base station that will be responsibis for providing wireless access after a handover; (b) re-routing all on-" going traffic to the new access point; (c) possibly tearing-down, and releasing unused VCs after a handover; and (d) buffering and synchronising to ensure cell sequencing and timeliness of ? arrival. Handover in WATM is regarded as the most important function to support terminal mobility.

(3) Mobile Location Management Protocol (MLMP) In a mobile network, a connection refers to two end-points that may migrate over time and be associated with different wireless access points. MLM protocols are thus enacted between MS, $\mathrm{BTS}$, and location servers.

(4) Mobile Routing Protocol (MRP)

Mobile routing has to support both the wired and wireless. segments. The wired segment refers to the path from a BTS to another BTS or end-host. The wireless segment refers to the wireless link from a BTS to an MS. An MS has to specify the destination host address. The source BTS will have to query the location server for the remote BTS address before routing packets/cells to this address. Hence, a two-level routing strategy is necessary.

(5) Mobile Media Access Control Protocol (MMACP) Since a BTS has to serve multiple MSs residing within its radio coverage, a media access control protocol is needed.

(6) Mobile Data-Link Control Protocol (MDLCP)

New types of flow control and retransmission schemes are 
required in mobile networks due to the intermittency of wireless links. In addition, such schemes should cater for different VCs having different $Q O S$ requirements [6, pp. 31-32].

\section{B. WATM Handover Requirements}

\section{(1) QoS Guarantee}

QOS for each WATM connection should be guaranteed during handovers. This is not easy because of the nature of radio connections.

(2) Resource Consumption Efficiency

Low buffering should be achieved during handover to avoid latency. Bandwidth consumption should be minimised.

\section{(3) Scalability}

The handover protocol should be able to serve as many MS handover requests as possible. It should also be feasible for wide geographic areas.

\section{(4) Low Latency}

The delays during handovers should be minimized to secure the QOS of WATM connections (audio/video).

\section{(5) Exclusive handover}

For a multimedia call, more than one connections (audio, video, and data) will exist at the same time. All the connections for the same WATM call should be handed over simultaneously.

\section{(6) Heterogeneous Mobile Connections}

In designing a handover protocol for WATM, both unicast and multicast connections should be supported. While most existing research works (e.g., Magic WAND, NEC, Radio ATM) have concentrated on unicast connections, multicasting, as an expected feature of ATM, should not be neglected in future research.

\section{(7) Exploitation of Radio Hints}

Signal strength detection can be used as an advance signal to trigger a handover well before an MS enters fully into the new wireless cell. Having a radio hint, however, means that the new BTS has to derive information about the MS's existing connections from the old BTS or other sources.

\section{A SOLUTION FOR WATM HANDOVER}

In this section, we describe a hybrid handover protocol that draws on experiences from the GSM network (Chapter 3) and meets the requirements for WATM handover.

\section{A. With-Radio-Hint Handover Protocol}

Control Flow

The With-Radio-Hint Handover Protocol (Figure 4) allows advance set-ups to be made. When MS receives the beacons from the new BTS (BTS NEW), after a certain signal threshold level, MS sends a handover hint(1) message to the current BTS (BTS OLD). As a result, BTS OLD sends BTS NEW a handover invoke(2) message containing MS's existing list of connections.



Figure 4. Control Flow Diagram for With-Radio-Hint Handover Protocol.

BTS NEW then determines if the handover is 'intra' or 'inter'. For intracluster (a wireless cluster is defined to be a collection of base transceiver stations which are connected to an ATM cluster switch) handovers, the cluster switch is the crossover switch (CX). Hence, no CX discovery is required.

However, if the next-cell beacon flag signifies an intercluster handover, the $\mathrm{CX}$ discovery process is invoked to compute the location of CX. New partial paths are then established from BTS NEW to CX. This is denoted by setup(3) and setup_ack(4). Hence, by the time MS enters well into the overlap region, the new partial paths have already been established. However, if the new partial paths cannot be established and BTS NEW does not receive the setup_ack(4) message, BTS NEW will time-out and invoke another CX discovery procedure to locate a different $C X$.

MS will now command BTS NEW to initiate a handover via the greet(5) message. This triggers BTS NEW to send a connection redirect(6) message to CX so that the ATM cells can now be rerouted over the new path. This mechanism avoids the 'sudden withdrawal' problem of MS: time-outs would abolish all the newly created partial paths if MS suddenly withdrew from entering the new cell. Once CX has performed the redirection, it asks BTS OLD to disconnect the old partial paths. If BTS OLD does not receive the tear-down(8a) message from $C X$, it will time-out. Likewise, if BTS NEW does not receive the greet(5) message from MS, it will time-out and the handover process is aborted. If the cell redirection operation is successful, a redirect_ack(7) message is returned to BTS NEW. BTS NEW then informs MS (via greet_ack(8)) the service parameters of the newly created partial connection. This facility allows the provision of QOS - MS is informed about the connection status and qualities of the wired links so that appropriate actions can be taken (such as degradation of QOS) by the mobile application.

Finally, MS returns BTS NEW a done(9) message indicating that it is now ready to receive and transmit data via BTS NEW. 
Due to the advance set-up and the use of buffering at the BTSs and $\mathrm{CX}$, handovers can now be performed quickly and seamlessly. To maintain ATM cell arrival sequence, one possibility is to return the done(9) message only after the 'endof-cell' marker is received by MS. If, for some reason, this indicator cell does not arrive within a time limit, the done(9) message will still be sent to BTS NEW to complete the handover process and cell loss or re-sequencing has to be handled by the higher layer protocols.

An exception occurs when MS sends the hint(1) message but, for some reason, this message is not received by BTS OLD and MS erroneously proceeds to issue a greet(5) message to BTS NEW. Since no new partial path has been established, the handover cannot be performed and no greet_ack(8) message will be returned to MS. MS can handle such exceptions by automatically invoking the Without-Radio-Hint Handover Protocol (Section V. B.).

\section{Message Flow}

Figure 5 explains the message flow in With-Radio-Hint Handover Protocol. Messages (8) and (8a) as well as (9) and (9a) can be performed in parallel. Except for messages (1), (5), (8) and (9), all messages occur over the wired ATM network.

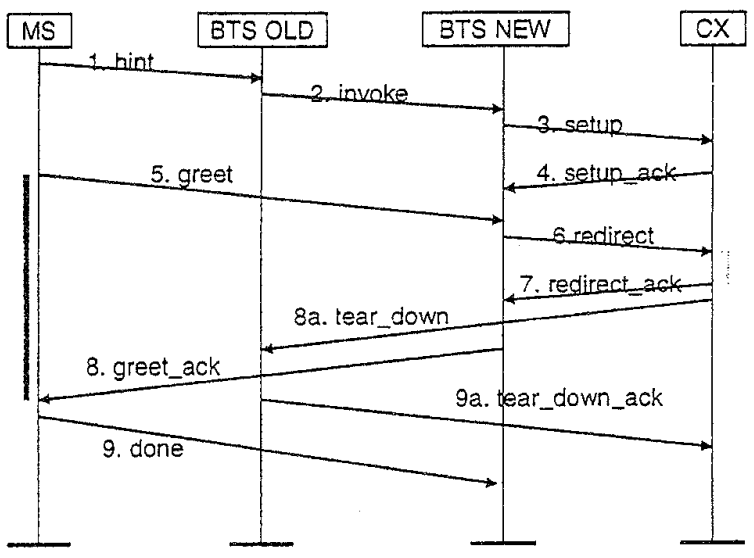

Downstream Disruption Time . Upstream Disruption Time

Figure 5. Message Flow Chart for With-Radio-Hint Handover Protocol.

\section{B. Without-Radio-Hint Handover Protocol}

\section{Control Flow}

Once a wireless link failure is detected, the migrating MS sends BTS NEW an explicit handover request, that is, the explicit_greet(1) message (Figure 6). Unlike the earlier greet(5), explicit_greet(1) contains sufficient information about MS's current connections. Hence, this design unavoidably incurs a penalty for using the wireless interface to convey the connection information of MS. Once BTS NEW receives explicit_greet(1), it invokes the CX discovery (for inter-cluster handovers) anc sends a partial path set-up(2) request message to $\mathrm{CX}$. Aftei receiving set-up_ack(3), BTS NEW immediately sends the connection redirect(4) request to $C X$, since the advance set-up is no longer present. When BTS NEW has received the redirect_ack(5) message from $C X$, it informs $M S$ about the re: connection by returning a greet_ack(6) message (similar tc greet_ack(8) in With-Radio-Hint Handover Protocol). Finally. MS sends a done(7) message to BTS NEW to signify its readiness to receive and transmit data.

BTS OLD can fail in two ways: (a) only its radio section fails to work or (b) the whole BTS fails. In the former case, the oid partial path tear-down process can still proceed. This tear-down process can occur in parallel with messages greet_ack(6) and done(7). In the latter case, however, BTS OLD will no longer respond to the tear-down(6a) message. But the connection between MS and BTS NEW has been established, thus ensuring that combined our two protocols work effectively.

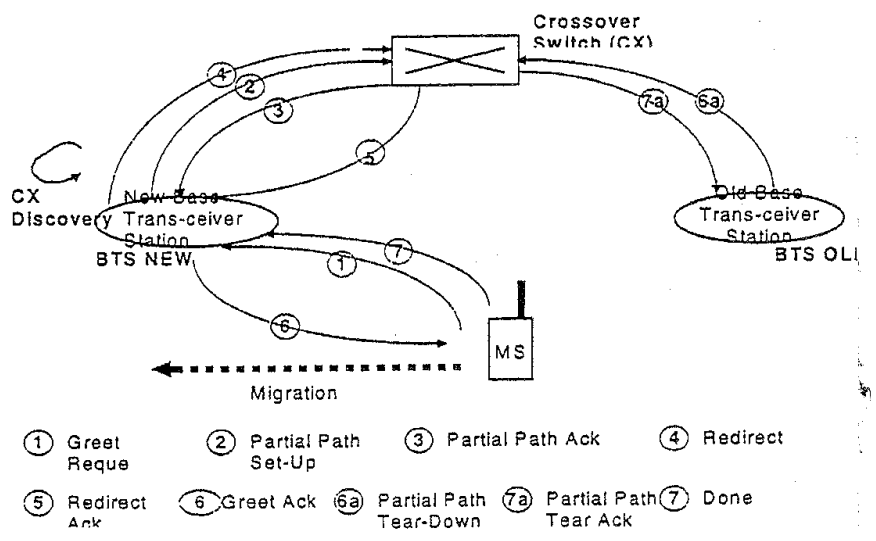

Figure 6. Control Flow Diagram for Without-Radio-Hint Handover Protocol Message Flow

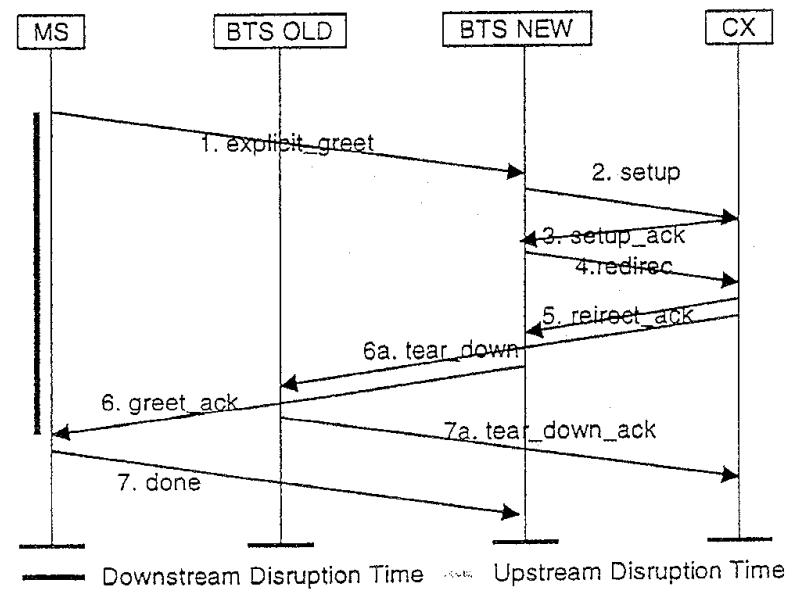

Figure 7. Message Flow Chart for Without-Radio-Hint Handover Protocol. 


\section{Summary}

The current ATM technology does not support handovers. Moreover, the existing handover schemes do not support multimedia streams. WATM handover thus becomes a crucial research issue. This chapter has presented a hybrid handover protocol that can fulfil these requirements. The protocol supports both intra- and inter-cluster handovers in a With- and WithoutRadio-Hint Wireless ATM network environments. Embedded into the handover protocol is a crossover switch discovery mechanism to support fast inter-cluster handovers with the consideration for mobile QoS. The capability of the handover protocol to support handovers of heterogeneous (e.g., both unicast and multicast) mobile connections has also been highlighted. Finally, the same handover protocol and crossover switch discovery scheme can also be used to support roaming between WATM networks.

\section{CONCLUSIONS AND FUTURE RESEARCH}

The current developments of Wireless ATM are mainly based on ATM as the backbone network with a "wireless-last-hop" extension to Mobile Stations. Mobility functions are being implemented into the ATM switches and/or base stations and WATM mobile users will be provided with multimedia services. This paper has contributed to the WATM research area by (1) focusing on Handover, one of the most important but relatively little studied features of WATM, (2) analysing design issues and requirements for Handover, and (3) revealing a hybrid WATM network handover solution.

Merwe [7] recently proposed that WATM could be enhanced by adopting an 'Open Multi-Service Network Architecture', where third party network services can be executed without the constraints. An extension of this concept has also resulted in proposals for 'programmable ATM switches' to support mobility. Mishra and Srivastava [8] argued that the implementations of various $\mathrm{VC}$ re-routing strategies are possible when the switches are controlled via remote connection managers.

Further research is needed to investigate how these and other developments affect the Handover solution proposed in this paper and how the solution can be tested and further improved and/or elaborated on by establishing a solid research agenda in the area of WATM Handover.

\section{REFERENCES}

[1] ITU-T Recommendation I.321, "B-ISDN Protocol Reference Model and Its Application". 1991.

[2] Händel, R., Huber, M.N., Schröder, S. "ATM networks Concepts, Protocols, Applications" $2^{\text {nd }}$ ed. Munich 1994, Addision-Wesley.

[3] The ATM Forum, "ATM User-Network Interface, Version 3.1 (UNI 3.1) Specification". 1994
[4] Mouly, M., Pautet, M.B., "The GSM system for Mobile Communications." Palaisseau, 1992.

[5] Rauhala, K., "Wireless ATM Presentation". Nokia Research Center, 1997.

[6] Toh, C-K., "Wireless ATM and AD-HOC Networks". Kluwer Academic Publishers, 1997

[7] Kobus Merwe, "Switchlets and Dynamic Virtual ATM Networks". Presentation Slides at Cambridge SRG Talk, March 1996.

[8] Partho P. Mishra \& Mani B. Srivastava, "Programmable ATM Switches For Mobility Support". In Proceedings of OPENSIG (Open signalling for Middleware \& Services Creation), April 1996. 